\title{
The Impact of Personalized Medicine on Pharmacovigilance
}

\section{Albert Wertheimer*}

Temple University, Philadelphia PA 19140, USA

As we all know, the field of pharmacovigilance has grown enormously in recent years. Some of this was caused by normal expansion at pharmaceutical companies and at biotechnology firms through the addition of new products and in pioneering fields. Perhaps the most compelling source of growth in the pharmacovigilance area has been through FDA requirements regarding the reporting of adverse events and other unexpected outcomes. There are many persons who see a continuing increase in the importance and size, especially in headcount, for a pharmacovigilance component of these companies. However, there is some reason to believe that new developments in the area of personalized medicine may change much of that expected progression. If we step back and think about the causes of adverse events and other untoward reactions to medications, we find that many of these are due to various metabolic pathways that differ both in group populations as well as within individuals.

If it persons were to be genotyped at birth and older people when they join a physicians practice, there would be sufficient information in most all circumstances to have the information and ability to select medications for that patient which should avoid some of the adverse drug effects, just as personalized medicine techniques should be able to determine what is the best medication selection for that individual patient from amongst all of those products having effectiveness in that therapeutic category. The same personalized medicine techniques can determine what enzymes are expressed in each individual and from the knowledge about the pharmacodynamics drug it be possible to avoid drugs which will compete for metabolism with the other drugs that that patient is taking and therefore greatly reduce the extent of toxicities from built-up quantities of those drugs which are slowly her and completely metabolized in individual patients. If this were to become a commonplace situation, it is very likely that the number of adverse events and the severity of those that continued would be vastly reduced, eliminating a great deal of pain suffering, as well as trauma and even morbidity while simultaneously saving money for the health insurers or managed care organizations.
It was interesting to note in an article in the Wall Street Journal on February 3 of this year how drug safety monitoring jobs have migrated to India. The article described how hundreds of workers sit at computer terminals inside buildings in the Bangalore area pouring over reams of medical records from the United States. The job is monitoring drug safety. The Journal reported that this is one of outsourcing's newest frontiers and how this new two billion-dollar business is booming. Perhaps the most astounding item in that article was the estimate that about 15,000 Indians work in the industry today compared with only about 200 in 2007 . The article went on to talk about drug safety but some of the other interesting features of the article or the fact that one company working there has tripled its drug safety work in the past seven years it was reported that they sent out around 600,000 reports of drug side effects to regulators last year.

To put this into full perspective, it is been estimated that adverse reactions to medication are the fourth leading cause of death in the United States, killing more than 100,000 people a year. Surely, these numbers can expect to fall even though it is unrealistic to assume that they will be eliminated as we become more adherent to personalized medicine methodologies and techniques.

For most persons, the benefits of personalized medicine have to do with clinical outcomes and economic savings as the most correct drug will be used the first time instead of the rather casual hit and miss system that we use today to eventually arrive at the most appropriate therapy. In addition to the impact on the field of pharmacovigilance which will be needed more than ever, even though there may be far fewer reports submitted each year. Headcounts will grow as more precise and detailed reporting requirements will be demanded by the FDA and as the standards for drug safety increase, probably shortening the time that a firm has to report serious adverse events to the FDA. As electronic health records become more ubiquitous, additional signals and patterns will be able to be identified very rapidly and persons in the pharmacovigilance field will be able to report in greater depth than me about adverse events, but will be able to report whether those adverse events are seen in subpopulation groups. These are exciting times.
*Corresponding author: Albert Wertheimer, Temple University, Philadelphia PA 19140, USA, Tel: 215-707-1291; Fax: 215-707-8188; E-mail: albertw@temple.edu

Received February 07, 2015; Accepted February 08, 2015; Published February 13, 2015

Citation: Wertheimer A (2015) The Impact of Personalized Medicine on Pharmacovigilance. J Pharmacovigilance 3: e133. doi:10.4172/2329-6887.1000e133

Copyright: ( 2015 Wertheimer A. This is an open-access article distributed under the terms of the Creative Commons Attribution License, which permits unrestricted use, distribution, and reproduction in any medium, provided the original author and source are credited. 